Historic, Archive Document

Do not assume content reflects current scientific knowledge, policies, or practices. 



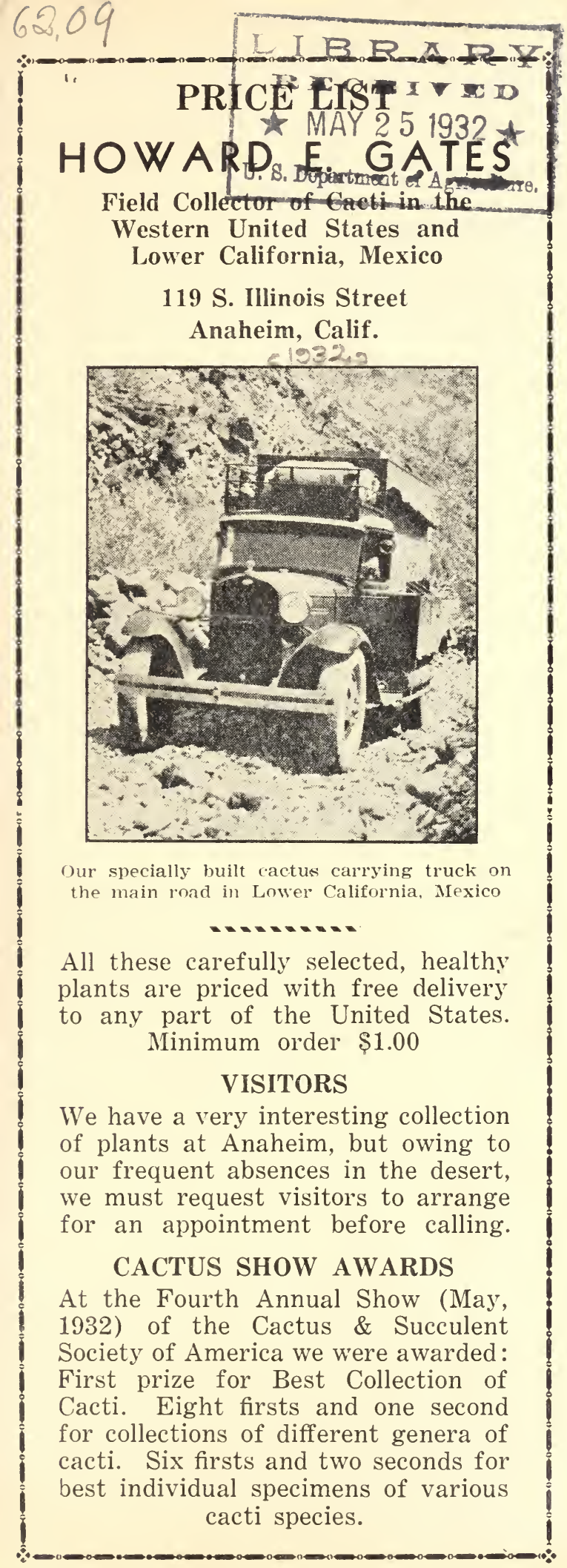




\section{GENERAL LIST}

Acanthocereus pentagonus-An attractive medium sized cereus type, 3 to 6 ribs

Carnegia gigantea - The Arizona Giant. Largest Cactus in the U. S. ........ Write us

Coryphantha deserti, Type "a"-A small biscuit shaped plant, white spines, sprinkled with straight dark ones, pink flower from Nevada $15,25,35,50 \mathrm{c}$

Coryphantha deserti, Type "b" - A little taller and whiter than Type "a". Salmon flowers, from Nevada $15,25,35,50 \mathrm{c}$

Coryphantha deserti, Type "c" c -Taller and darker than the above forms. The true deserti from California

$15,25,35,50 \mathrm{c}$

Coryphantha recurvata-Very large round heads, yellow recurved spines, yellow flower $35,50,75, \$ 1.00$

Coryphantha, new species - Large single headed plants, white spines inter mingled with dark ones. Large pink flower.

From Utah $25,50,75 \mathrm{c}$

Echinocactus polycephalus - Large heads with long gray spines.

Singles ......25, 35, 50c. Clusters 75c up

Echinocereus engelmannii-A cluster cactus with vari-colored straight spines. Magnificient large magenta flower.

Singles 15, 25c. Clusters 35c up

Echinocereus fendleri - A very attractive large pink flowered form, from Arizona $25,35,50 \mathrm{c}$

Echinocereus mojavensis-Forms clusters of large green "pickles" with white or gray spines. Scarlet flower.

Singles ..............15, 25c. Clusters 35c up

Echinocereus octacanthus-Forms clusters of smaller, more compact heads than Mojavensis. Red flower. From Utah.

Singles ..............15, 25c. Clusters, 35c up

Echinocereus rigidissimus - The beautiful Arizona rainbow cactus ...........25, 35, 50c

Ferocactus acanthodes - The red spined, California barrel cactus.

Yellow flowers $25,35,50,75$ c up

Ferocactus colvillei - An Arizona beauty. Heavy spines 
Ferocactus johnsonii - A small round straight spined plant with many reddish straight spines. Flower pink with red blotches $15,25,35,50$ c

Ferocactus johnsonii, dark form-Another species or a soil variation. Lavender gray spines. Yellow flower ........15, 25, 35, 50c

Ferocactus viridescens-The squat, heavy spined nigger head from San Diego

County 15, 25, 35c

Hamatocactus setispinus - An attractive, small, short spined cactus from Texas. Many beautiful yellow flowers followed by red fruit $15,25,35$ c

Harrisia martini-A slender climbing cactus with a very large white blossom. Fine to use as a grafting stock $50,75, \$ 1.00$

Lemaireocereus thurberi - A pipe organ cactus. A large upright cereus type. Plants from one to five feet $\$ 2.50$ per foot

Lophophora williamsii-The peyote or mescal button. Contains a narcotic. Used in Indian ceremonials. Spineless. Very strange $25,35 \mathrm{c}$

Neomammillaria dioica-White spines, few dark hooked spines, forms

clusters

15, 25, 35c

Neomammillaria grahami - White spines, few dark hooked spines, pink flower, red fruit

$15,25,35 \mathrm{c}$

Neomammillaria heyderi-A flat, milky type, white spines, white flower, red fruit

$15,25,35 c$

Neomammillaria johnstonii - Rare. Flat milky type, red spines, yellowish

flower

$25,50,75 c$

Neomammillaria macdougalii-Larger than Heyderi. White spines ...........15, 25, 35c

Opuntia arbuscula-Forms a compact bush of small branches ....................15, 25, 35c

Opuntia basilaris-(Beaver tail). Purplish pads, very minute spines, magnificent magenta flower. Very showy. Easy to grow

$15,25,35 \mathrm{c}$

Opuntia basilaris-Yellow flowered form, 25c Opuntia new species-Of Basilaris group, long spines, pink flower, from Utah, 25c Opuntia chlorotica-A large upright type, blue pads, slender drooping yellow spines. Quite rare 50,75 c up 
Opuntia erinacea-The pink spined grizzly bear cactus. Yellow flower ........15, 25 35c

Opuntia leptocaulis - A miniature tree. Bright red fruit in winter ........15, 25, 35c

Opuntia ramosissima - Many small woody branches

$25,35,50 \mathrm{c}$

Opuntia Santa Rita-Upright, blue pads, nearly spineless, yellow flower ....35, 50c Opuntia spinosior-Attractive green stemmed cholla. Purple flower ........15, 25, 35c

Opuntia treleasii-One of the basilaris group with many small spines $15,25,35 \mathrm{c}$ Opuntia ursina - The long white spined grizzly bear cactus. Pink flower, 25, 35, 50c

Phellosperma tetrancistra-Resembles Neo. grahami, but rare. Large pink

flower

$25,35,50 \mathrm{c}$

Sclerocactus whipplei-Hooked spined, pink flowered species from Utah ........35, 50, 75c

All of the above plants are delivered free within the United States when the order amounts to $\$ 1.00$ or more. We will gladly quote prices on large specimen plants upon request. We label one of every species in each shipment. We are receiving a pleasing number of repeat orders and we are rapidly building up a large clientele of satisfied customers.

\section{PLANTS FROM LOWER CALIFORNIA, MEXICO}

These plants are propagated from our own collections in Lower California, in which territory we have had a wider experience than any other collector. Our collection of these species is the best in the United States. Prices on these are higher than prices on local species owing to the great cost in time, money and health it has required to assemble our stock of mother plants.

Bartschelia schumannii-Forms low clusters of small white spined heads, beautiful pink flower, red fruit $\$ 1.50$

Cochemeia halei-Forms clusters, straight purplish spines, scarlet flower shaped like a Zygocactus. Very rare $\$ 2.00$

Cochemeia poselgeri-A wonderful but little known plant 
Echinocereus brandegeeii-A tall headed, straight spined cluster plant, purplish flowers

Echinocereus barthelowanus-Short heads, very compact clusters, purplish

spines

Echinocereus maritimus-Short heads, very compact clusters, beautiful yellow

flower

Echinocereus pensilis-One of our rarest species Write us

Echinocereus sciurius-Small, white spined mammillaria like clusters. Large pink flower

Grusonia new species-Our own discovery. Second species known of this genus. See Opuntia No. 131, July, 1931 Cactus \& Succulent Journal $\$ 2.50$

Lemaireocereus littoralis-A dwarf lemaireocereus. Very rare. See July, 1931, Cactus \& Succulent Journal ........Write us

Lophocereus schottii-18 inch rooted, hairy, tip cuttings $\$ 7.50$

Lophocereus schottii monstrosa, sargentianus and australis - Illustrated August, 1931, and February, 1932, Cactus \& Succulent Journals

Write us

Machaerocereus eruca-The creeping devil cactus

Write us

Neomammillaria la pacena-A new species. See Feb., 1932 "Desert Magazine", \$1.50

Neomammillaria No. 68 - New species, slightly resembling $N$. fasciculata but larger. Pink flowers, red fruit

$\$ 1.50$

Neomammillaria No. 128-See August, 1931, Cactus \& Succulent Journal

$\$ 1.50$

Peniocereus johnstonii - An exceedingly rare tuberous rooted cactus. ...... Write us

Pereskiopsis porteri-Leafy climber, yellow flower, beautiful long orange colored fruit. Seedlings over six inches high

$50 \mathrm{c}$

Pereskiopsis gatesii-New species. See Feb. 1932, Cactus \& Succulent Journal .... $\$ 1.00$

Opuntia burrageana-Slender cylindric type, many dark short spines

$\$ 1.00$

Opuntia cholla-The cylindric species properly named "Cholla"

$\$ 1.00$

Opuntia ciribe - Beautiful white spined cylindric species 
Opuntia gosseliana - Beautiful, green flat padded opuntia, yellow flower ........\$1.50

Opuntia invicta - Grows like an Echinocereus. Yellow flower and fruit. Write us

Opuntia tesajo-Small, woody, branching, yellow flowered species. Rare .........\$1.00

Opuntia No. 95. New species-Very slender cylindric type. Red fruit ..............\$1.00

Opuntia No. 117, possibly clavelliana-Long joints, long spines, bronze flowers, cylindric

$\$ 1.00$

Opuntia No. 136, new species-Blue padded, white spined, platyopuntia. See Tuna Morada, July, 1931, Cactus \& Succulent Journal

$\$ 1.50$

Opuntias No. 141, 145, 151-Un-identified cylindric species $\$ 1.00$

Wilcoxia striata-Another very rare plant. Grafted plants Write us

Pedilanthus macrocarpus, a Euphorbia Produces many tall, smooth, leafless, rod like branches topped with red

flowers $\$ 1.50$

If you wish other very rare species write to us about our next collecting trip into Lower California, Mexico.

\section{THE CACTUS SOCIETY}

The three dollar annual membership fee of the Cactus \& Succulent Society of America will bring you the monthly Cactus \& Succulent Journal as well as many other advantages. Margaret Kincher, 1421 Dominion Ave., Pasadena, Calif., is Secretary.

"The Personal Tale of a Cactus Collector" tells of our adventures in Lower California, Mexico in 1930. We send it for 25c.

The "Desert Magazine," Box 68, Pasadena, California., \$1.50 per year is a very interesting monthly.

"The Cactus Book" by Dr. Houghton, $\$ 2.25$, tells you how to grow cactus. At your bookstore. 

